\title{
Correlation between mood and heart rate variability indices during daily life
}

\author{
Kohzoh Yoshino ${ }^{1 *}$, Katsunori Matsuoka ${ }^{2}$ \\ ${ }^{1}$ Health Research Institute, National Institute of Advanced Industrial Science and Technology (AIST), Osaka, Japan; \\ *Corresponding Author: yoshino-k@aist.go.jp \\ ${ }^{2}$ Life Science and Biotechnology, National Institute of Advanced Industrial Science and Technology (AIST), Ibaraki, Japan.
}

Received 1 June 2011; revised 20 July 2011; accepted 17 August 2011.

\begin{abstract}
We investigated the correlation between mood and heart rate variability (HRV) indices during daily life. The RR-interval and body acceleration of 40 normal male subjects were recorded using ambulatory device for 48 to 72 hours. Every hour that the subjects were awake they registered their current mood on a Visual Analogue Scale questionnaire. The questionnaire scales eight of the subjects' current moods. Those are happiness, tension, fatigue, worry, depression, anger, vigor, and confusion. The following four HRV indices were calculated. Those are heart rate, root mean square of successive differences of RR-interval sequence, the normalized high-frequency $(0.15-0.4 \mathrm{~Hz})$ power of RR-interval variability, and mean frequency in the high-frequency band of RR-interval variability. The calculated HRV indices data and the mood data were normalized individually, the data with body acceleration exceeding $30 \mathrm{mG}$ were excluded from the analysis to reduce the effect of exercise, and the differences from the first day ( $\Delta$ mood and $\Delta \mathrm{HRV}$-index) were taken to reduce the effect of circadian rhythm. The most three highly correlated combinations were $\Delta$ vigor and $\Delta$ HFnu $(R=-0.24, p<0.0001), \Delta$ vigor and $\triangle$ RMSSD $(R=-0.24, p<0.0001)$, and $\Delta$ vigor and $\Delta \mathrm{HR}(R=0.22, p<0.001)$. Vigor exhibited the most significant correlations with HRV indices of eight moods.
\end{abstract}

Keywords: Mood; Autonomic Nervous System; Ambulatory; Heart Rate; Emotion

\section{INTRODUCTION}

It is important for mental health management to de- velop a method that can assess mood during daily life in stressful modern society. Three methods have been proposed from mental state assessment. The first method is based on subjective self-evaluation. The second is based on biochemical analysis that quantifies the concentration of stress-related substrates in blood plasma, urine, and saliva. The third method is based on variability analysis of physiological signals reflecting the state of the autonomic nervous system. The third method has the following advantages for mental state monitoring compared with the other two. 1) It can be measured continuously with a short sampling interval; 2) it does not interrupt daily life activities; 3 ) it can compute (assess) the values of indices automatically; 4) it is less affected by psychological bias than the subjective self-evaluation method.

Heart rate variability (HRV) is a representative physiological signal reflecting states of the autonomic nervous system that can be continuously measured during daily life with a short time interval. In order to develop a method that can assess mood states from $H R V$, it is necessary to understand how mood and HRV during daily life are correlated [1]. Several studies have investigated the relationship between stress or mood and heart rate during daily life. Dobkin and Pihl [2] demonstrated that the heart rate of normal subjects during daily life is significantly higher when stressed than when not stressed. They also revealed that anxiety and hostility contributes to the heart rate elevation. Langewitz et al. [3] found that the perceived stress level is significantly correlated with heart rate in normal subjects. Johnston and Anastasiades [4] reported that heart rate during daily life is related to arousal, stress, and time pressure in very few normal subjects. Shapiro et al. [5] reported no correlation of mood with heart rate. Sloan et al. [6] showed that the LF/HF ratio (sympathovagal balance index) is significantly correlated with stress (irritable, tense, pressured, and less happy) during daily life in normal subjects. Yoshino and Matsuoka [7] found that both daily depression and worry shift toward sympathetic 
dominance during subsequent sleep. Bacon et al. [8] reported the significant association of high levels of negative emotions (anger, stress, and sadness) with decreases in LF and HF powers, but higher levels of positive emotion with increases in LF power in patients with coronary artery disease. Schwerdtfeger et al. [9] showed that depression was related to higher heart rate throughout the day. Moreover, there was a tendency toward lower HRV in individuals with higher depression scores, and this association was moderated by social context.

However, to our knowledge, no studies have investigated the relationship between HRV indices and various moods including vigor in normal subjects during daily life. In this study, we analyzed the correlation between four HRV indices and eight moods including vigor in normal male subjects during daily life.

\section{METHODS}

Normal healthy male subjects $(N=40$, age: 20 to 39 years) participated in this experiment. None of the subjects had a history of cardiovascular, respiratory, or brain disease and none were currently using any medications. All subjects provided their informed written consent as approved by the Ethical Committee on Human Research at National Institute of Advanced Industrial Science and Technology. Subjects intermittently wore an ambulatory device Active tracer (AC-301, GMS, Japan) in a pouch at the waist for a total of 48 to 72 hours during daily life, including sleep, except when taking a bath or shower. This device records the RR-intervals of the electrocardiogram and body acceleration.

Every hour that the subjects were awake they registered their current mood on a 100-mm-long Visual Analogue Scale (VAS) questionnaire on which the endpoints were labeled "lowest" and "highest". The questionnaire scales eight of the subjects' current moods: happiness, tension, fatigue, worry, depression, anger, vigor, and confusion. We instructed subjects not to answer the questionnaire more than 15 minutes after the predetermined time, and we strictly prohibited subjects from recalling a past mood when answering the questionnaire.

The following four HRV indices 1) to 4) were calculated from RR-interval sequence data collected during $10 \mathrm{~min}$ before answering the VAS questionnaires if at least $96 \%$ of 10-min RR-interval records were within valid range $(0.3-2.0 \mathrm{sec})$. The RR-intervals out of this range were omitted from the analysis. If the mean body acceleration in the 10 -min exceeded $30 \mathrm{mG}$, the corresponding RR-interval data were excluded from the analysis in order to exclude the effect of body movement. It has been reported that $157.5 \mathrm{mG}$ is the optimum value of threshold to discriminate activity state and rest by body acceleration [10]. We used the value $30 \mathrm{mG}$ which is five times smaller than it in this study. The inter-individual mean excluded ratio was $47.2 \% \pm 12.5 \%$, and there was no significant difference between before and after noon.

1) Heart rate (HR) and 2) root mean square of successive differences (RMSSD) of RR-interval sequence were calculated for each 10-min data set. HR takes low values and RMSSD takes high values when the balance of autonomic nervous system activity (sympathovagal balance) shifts toward parasympathetic dominance.

The following frequency analysis was performed in addition to the time domain analysis. The power spectrum was calculated by applying a fast Fourier transform (FFT) to the RR-interval signal for each 10-min data set. The area (i.e., power) of high-frequency components (HF: 0.15 to $0.4 \mathrm{~Hz}$ ) of the power spectrum was divided by the total power from which the very-low-frequency component (VLF: $<0.04 \mathrm{~Hz}$ ) had been subtracted in order to calculate (3) the normalized HF power (HFnu), which takes higher values when the sympathovagal balance shifts toward parasympathetic dominance. 4) Mean frequency in HF band of the power spectrum (MFHF) were calculated. MFHF is related to respiratory frequency.

To reduce inter-individual variability, we normalized the measured values of mood level and HRV indices by dividing by their amplitudes (maximum-minimum in measurement) after subtracting their minimum values for each subject. Moreover, to reduce the effect of circadian rhythm, the differences of the normalized values of mood level and HRVI indices on the second or third day from those at the same time of the first (reference) day (denoted as $\Delta$ mood and $\Delta$ HRVI) were calculated for each subject.

Before applying the correlation analysis, we confirmed that the inter-individual differences of $\Delta$ mood and $\triangle H R V I$ variations were not relatively high. This was done by comparing the inter-individual means and standard deviations of $\Delta$ mood and $\Delta H R V I$ variations. Following the confirmation, Pearson's correlation coefficients between eight $\Delta$ moods and four $\Delta$ HRVIs were calculated.

\section{RESULTS}

The comparison results are summarized in Table 1. The most three highly correlated combinations were $\Delta$ vigor and $\Delta$ HFnu $(R=-0.24, p<0.0001)$ (Figure 1 right), $\Delta$ vigor and $\Delta \operatorname{RMSSD}(R=-0.24, p<0.0001)$, and $\Delta$ vigor and $\Delta \mathrm{HR}(R=0.22, p<0.001)$ (Figure 1 left). There were three combinations whose $p$-values were less than 0.001 (Table 1). All of them were combinations with $\Delta$ vigor. This indicates that vigor has the 
Table 1. Pearson's correlation coefficients between four $\Delta$ HRVIs and eight $\Delta$ moods.

\begin{tabular}{|c|c|c|c|c|c|c|c|c|}
\hline & $\Delta$ happiness & $\Delta$ tension & $\Delta$ fatigue & $\Delta$ worry & $\Delta$ depression & $\Delta$ anger & $\Delta$ vigor & $\Delta$ confusion \\
\hline$\Delta \mathrm{HR}$ & 0.11 & 0.05 & -0.02 & 0.05 & 0.05 & 0.01 & $0.22 * *$ & 0.03 \\
\hline$\Delta \mathrm{RMSSD}$ & $-0.19^{*}$ & -0.07 & 0.08 & -0.03 & 0.00 & -0.01 & $-0.24 * * *$ & -0.05 \\
\hline$\Delta$ HFnu & -0.03 & -0.13 & -0.07 & -0.05 & -0.04 & -0.10 & $-0.24 * * *$ & -0.10 \\
\hline$\triangle \mathrm{MFHF}$ & -0.12 & -0.08 & -0.11 & -0.06 & -0.01 & 0.01 & -0.12 & -0.00 \\
\hline
\end{tabular}

$*: p<0.01, * *: p<0.001, * * *: p<0.0001$.
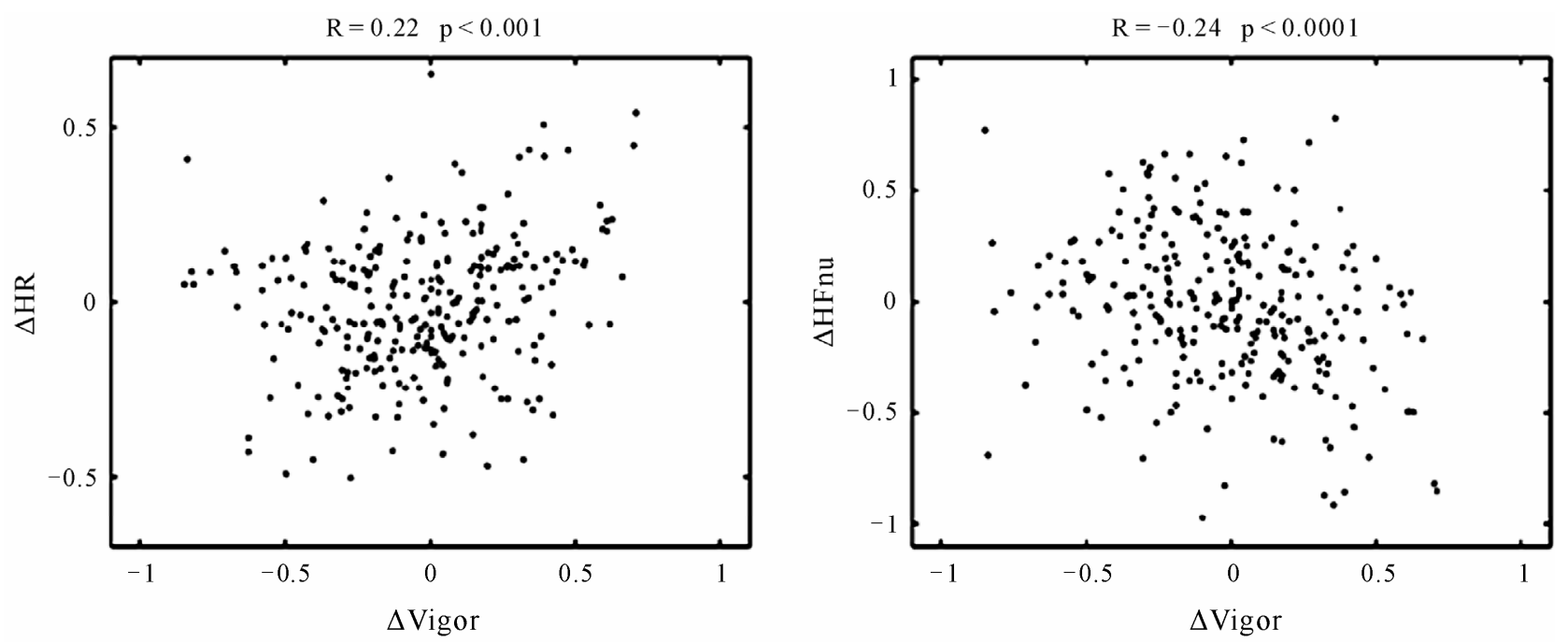

Figure 1. Scatter plots of $\Delta$ vigor versus $\Delta H R$ (left) and $\Delta$ vigor versus $\Delta H F n u$ (right).

most significant correlations with HRV indices of eight moods.

\section{DISCUSSION}

The significant negative correlations between $\Delta$ vigor and $\Delta$ HFnu $(R=-0.24, p<0.0001)$ and between $\Delta$ vigor and $\triangle \mathrm{RMSSD}(R=-0.24, p<0.0001)$ and the significant positive correlation between $\Delta$ vigor and $\Delta \mathrm{HR}(R=$ $0.22, p<0.001$ ) suggest that vigor during daily life shifts the autonomic nervous system balance toward sympathetic dominance.

Russell proposed a circumplex model that defines emotion space by pleasure-displeasure (valence) axis and arousal-sleep (arousal) axis [11]. Thayer decomposed the arousal axis into two dimensions based on the results from factor analysis $[12,13]$. One is tense arousal and the other is energetic arousal. The tense arousal represents a continuum from calmness to anxiety, whereas the energetic arousal reflects a continuum from tiredness to energy. Dickman broke down the energetic arousal still further into two dimensions of wakefulness and vigor [14]. The result from the presented study implies that autonomic nervous system (HRV) indices covariate with vigor the fourth dimension axis in emotion space during daily life.

In our previous study, we demonstrated that both daily depression and worry shift toward sympathetic dominance during subsequent sleep, whereas vigor had little effect on the autonomic nervous system balance during subsequent sleep [7]. The results in this study and in our previous study imply that vigor during daily life concurrently has a relatively strong effect on shifting the autonomic nervous system balance toward sympathetic dominance, but the effect is not sufficiently long-lasting to make an effect during subsequent sleep.

There are several limitations pertain to this study. First, the effects of circadian rhythm on mood and HRVI have not been completely removed, although we took the differences of the values of indices on the second or third day from those at the same time of the first (reference) day. This is because of the day-to-day variation in the circadian rhythm of mood and autonomic nervous activity.

Second, the result of this study can be applied to only half of the daily awaking time. This is because about half $(47.2 \%)$ of the case was excluded from the analysis, since the mean body acceleration was over $30 \mathrm{mG}$.

In summary, we demonstrated that vigor during daily 
life has the most significant correlations with HRV indices (autonomic nervous system balance) of eight moods.

\section{ACKNOWLEDGEMENTS}

This work was supported by Grant-in-aid for Scientific Research (KAKENHI) for Young Scientists (B) (No.19700557) from the Ministry of Education, Culture, Sports, Science, and Technology, Japan.

\section{REFERENCES}

[1] Kreibig, S.D. (2010) Autonomic nervous system activity in emotion: A review. Biological Psychology, 84, 394421. doi:10.1016/j.biopsycho.2010.03.010

[2] Dobkin, P.L. and Pihl, R.O. (1992) Measurement of psychological and heart rate reactivity to stress in the real world. Psychotherapy and Psychosomatics, 58, 204-214. doi:10.1159/000288629

[3] Langewitz, W., Ruddel, H. and Von Eiff, A.W. (1987) Influence of perceived level of stress upon ambulatory blood pressure, heart rate, and respiratory frequency. Journal of Clinical Hypertension, 3, 743-748.

[4] Johnston, D.W. and Anastasiades, P. (1990) The relationship between heart rate and mood in real life. Journal of Psychosomatic Research, 34, 21-27. doi:10.1016/0022-3999(90)90004-N

[5] Shapiro, D., Jamner, L.D. and Goldstein, I.B. (1997) Daily mood states and ambulatory blood pressure. Psychophysiology, 34, 399-405. doi:10.1111/j.1469-8986.1997.tb02383.x

[6] Sloan, R.P., Shapiro, P.A., Bagiella, E., Boni, S.M., Paik, M., Bigger, J.T. Jr., Steinman, R.C. and Gorman, J.M. (1994) Effect of mental stress throughout the day on car- diac autonomic control. Biological Psychology, 37, 89-99. doi:10.1016/0301-0511(94)90024-8

[7] Yoshino, K. and Matsuoka, K. (2009) Effect of mood during daily life on autonomic nervous activity balance during subsequent sleep. Autonomic Neuroscience, 150, 147-149. doi:10.1016/j.autneu.2009.03.013

[8] Bacon, S.L., Watkins, L.L., Babyak, M., Sherwood, A., Hayano, J., Hinderliter, A.L., Waugh, R. and Blumenthal, J.A. (2004) Effects of daily stress on autonomic cardiac control in patients with coronary artery disease. American Journal of Cardiology, 93, 1292-1294. doi:10.1016/j.amjcard.2004.02.018

[9] Schwerdtfeger, A. and Friedrich-Mai, P. (2009) Social interaction moderates the relationship between depressive mood and heart rate variability: Evidence from an ambulatory monitoring study. Health Psychology, 28, 501-509. doi:10.1037/a0014664

[10] Mathie, M.J., Coster, A.C.F., Lovell, N.H. and Celler, B.G. (2003) Detection of daily physical activities using a triaxial accelerometer. Medical \& Biological Engineering \& Computing, 41, 296-301. doi:10.1007/BF02348434

[11] Russell, J.A. (1979) Affective space is bipolar. Journal of Personality and Social Psychology, 37, 345-356. doi:10.1037/0022-3514.37.3.345

[12] Thayer, R.E. (1967) Measurement of activation through self-report. Psychological Reports, 20, 663-678.

[13] Thayer, R.E. (1978) Factor analytic and reliability studies on the Activation-Deactivation Adjective Checklist. Psychological Reports, 42, 747-756.

[14] Dickman, S.J. (2002) Dimensions of arousal: Wakefulness and vigor. Human Factors, 44, 429-442. doi:10.1518/0018720024497673 